\title{
Three-phase frequency measurement under non-sinusoidal conditions using the static reference frame
}

\author{
Mañana, M.; Ortiz, A.; Pérez, S.; Renedo, C. and Eguíluz, L.I. \\ Department of Electrical Engineering \\ E.T.S.I.I.T, University of Cantabria \\ Avda. Los Castros s/n. Santander 39005 Cantabria (Spain) \\ phone:+34 942201378, fax:+34 942201385, e-mail: mananam@unican.es
}

\begin{abstract}
The utilization of Clark's transformation in the estimation of the power system frequency provides more robustness to the classical single-phase methods. One of the advantages of this technique is that extract the frequency information contained in the three-phase system. This is particularly important when asymmetric sag generates a zero voltage in one of the three phases.
\end{abstract}

\section{Key words}

Frequency measurement, Power quality.

\section{Introduction}

Frequency measurement is already an important topic in power systems because it reflects the dynamic energy balance between the generation and the consumption. Distributed generation in weak coupling points of the distribution networks can be the origin of frequency deviation that should be carefully controlled. Some nonlinear loads, such as arc furnaces, ASD and other power electronics devices are sources of harmonics and high frequency noise. In addition, the frequency of a distribution network can vary extremely fast during transient events so it can be very difficult to track the frequency with enough accuracy.

In spite of the fact that there are many digital algorithms used to estimate frequency, almost all of them are based on the measurement of a single phase of the system, so the algorithms exhibit poor behavior when the tracked phase suffers a dip or a transient. Some instrumentation manufacturers have solved the problem registering the frequency measured in each phase. The problem with this single phase approach is that standard regulations ask for the system frequency so the utilization of a single phase should be tagged in order to know what phase is being used.
The proposed technique uses the information contained in the three phases in a simultaneous way. This approach increases the robustness of the measurement technique, which can track the frequency in case of lack of one arbitrary phase (dips, notches or small interruptions).

The organization of this paper is as follows: we describe in detail the transformation technique in Sections 2 and 3. After that, Section 4 summarizes some tests run under sinusoidal and non-sinusoidal conditions, such as high frequency noise, harmonic distortion and transient during a short circuit. Finally, we give some conclusions in Section 5.

\section{Frequency Algorithm Under Sinusoidal Condition}

The three-phase voltage has a digital representation that it is assumed to have the form:

$$
\begin{aligned}
& x_{a}(n)=X_{a} \cos \left(n \omega T+\varphi_{a}\right) \\
& x_{b}(n)=X_{b} \cos \left(n \omega T+\varphi_{b}\right) \\
& x_{c}(n)=X_{c} \cos \left(n \omega T+\varphi_{c}\right)
\end{aligned}
$$

where $\mathrm{T}$ is the sampling period.

Equations (1a), (1b) and (1c) can be put into the following matrix formulation,

$$
\left[\begin{array}{l}
x_{0}(n) \\
x_{\alpha}(n) \\
x_{\beta}(n)
\end{array}\right]=\frac{2}{3}\left[\begin{array}{ccc}
\frac{1}{2} & \frac{1}{2} & \frac{1}{2} \\
1 & \cos \left(\frac{2 \pi}{3}\right) & \cos \left(-\frac{2 \pi}{3}\right) \\
0 & \sin \left(\frac{2 \pi}{3}\right) & \sin \left(-\frac{2 \pi}{3}\right)
\end{array}\right]\left[\begin{array}{l}
x_{a}(n) \\
x_{b}(n) \\
x_{c}(n)
\end{array}\right]
$$


$\mathbf{x}_{0 \alpha \beta}=\left[\begin{array}{c}x_{0}(n) \\ x_{\alpha}(n) \\ x_{\beta}(n)\end{array}\right]=\frac{2}{3}\left[\begin{array}{ccc}1 & 1 & 1 \\ 2 & 2 & 2 \\ 1 & -\frac{1}{2} & -\frac{1}{2} \\ 0 & \frac{\sqrt{3}}{2} & -\frac{\sqrt{3}}{2}\end{array}\right]\left[\begin{array}{c}x_{a}(n) \\ x_{b}(n) \\ x_{c}(n)\end{array}\right]=\mathbf{C} \cdot \mathbf{x}_{a b c}$

where $\mathbf{C}$ is the well-known transformation introduced by Clark [1], which transforms the three-phase voltage variables into the zero-sequence and direct and quadrature-axis components. Both $\mathbf{C}$ and its inverse $\mathbf{C}^{-1}$ are widely used in the generalized theory of electrical machines [2,3]. When it is necessary to use a powerinvariant form, the terms $2 / 3$ and $1 / 2$ are replaced by $\sqrt{2 / 3}$ and $1 / \sqrt{2}$ respectively. In our approach to the problem it doesn't matter if the matrix transformation is power-invariant or not. From a graphical point of view, $x_{\alpha}(n)$ and $x_{\beta}(n)$ are the rectangular coordinates of a point that is changing with time at a rate that it is proportional to the system frequency. Under sinusoidal conditions, equations (1a)-(1c) have the same amplitude,

$X_{a}=X_{b}=X_{c}=X$

In addition, the components are symmetrical so,

$\varphi_{b}=\varphi_{a}+\frac{2 \pi}{3}$

$\varphi_{c}=\varphi_{a}-\frac{2 \pi}{3}$

Considering that $\varphi_{a}=0$ and substituting equations (1a)(1c) in (2)

$$
\left[\begin{array}{c}
x_{0}(n) \\
x_{\alpha}(n) \\
x_{\beta}(n)
\end{array}\right]=\left[\begin{array}{c}
0 \\
X \cos (n \omega T) \\
X \cos \left(n \omega T+\frac{\pi}{2}\right)
\end{array}\right]
$$

The point defined by the coordinates $\left(x_{\alpha}(n), x_{\beta}(n)\right)$ has a ratio equal to $\mathrm{X}$ and an angular velocity of $\omega$,

$\omega=2 \pi f$

The angular velocity can be also computed graphically. If we consider two consecutives samples of the $\alpha \beta$-vector, $\mathrm{x}_{\alpha \beta}(\mathrm{n})$ and $\mathrm{x}_{\alpha \beta}(\mathrm{n}-1)$ then, the angular difference $\Delta \theta(n)$ between the two vectors can be approximated as,

$$
\begin{aligned}
\Delta \theta(n)= & \theta(n)-\theta(n-1) \cong \\
& \frac{\sqrt{\left(x_{\alpha}(n)-x_{\beta}(n-1)\right)^{2}+\left(x_{\alpha}(n)-x_{\beta}(n-1)\right)^{2}}}{X}
\end{aligned}
$$

Equation (7) considers that $\Delta \theta(n)$ is small enough.
Finally, the frequency $f(n)$ of the input signal is

$$
f(n)=\frac{\Delta \theta(n)}{2 \pi T}
$$

where $T$ is the time needed to reach $x_{\alpha \beta}(n)$ from $x_{\alpha \beta}(n-1)$. Figure 1 summarizes the proposed method. The smallest the sampling period $\mathrm{T}$ is, the smallest the estimated frequency error will be.

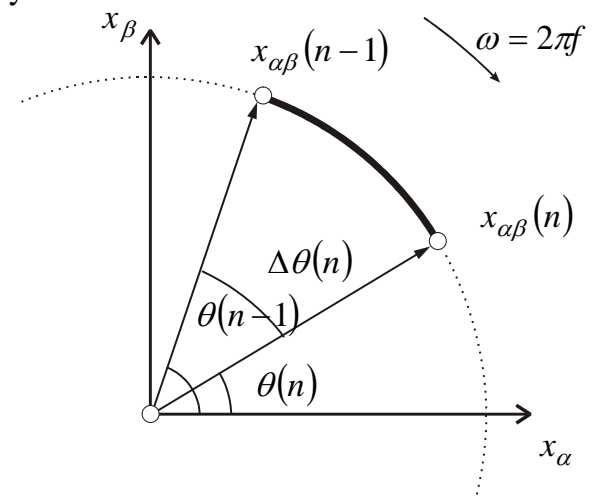

Fig 1. Static reference frame representation.

\section{Frequency Algorithm Under Non- Sinusoidal Conditions}

Equation (8) provides good frequency estimations when the voltage exhibits small distortion levels. However, the computer frequency shows poor accuracy under sags and swells.

One way to increase the performance of the procedure is to introduce a feedback for computing the frequency in a recursive fashion.

The proposed method is based on a PID controller. The procedure starts from an arbitrary value, which is usually set to a frequency of $50 \mathrm{~Hz}$. At each step the new frequency value is computed using the last value and an approximation that is computed considering an ideal trajectory of the vector defined by coordinates $\left(x_{\alpha, t}(n), x_{\beta, t}(n)\right)$, where,

$\mathrm{x}_{\alpha, \mathrm{t}}(\mathrm{n}) \alpha$ component of an ideal sinusoidal voltage signal equal to the fundamental component of $x(n)$.

$\mathrm{x}_{\beta, t}(\mathrm{n}) \beta$ component of an ideal sinusoidal voltage signal equal to the fundamental component of $x(n)$.

The points defined by vectors $\mathbf{x}_{\alpha \beta}(n)$ and $\mathbf{x}_{\alpha \beta, t}(n)$ are the same under sinusoidal conditions. If we consider a real signal $\mathbf{x}_{a b c}(n)$, the estimated $\mathbf{x}_{0 \alpha \beta, t}(n)$ at any arbitrary time step $\mathrm{n}_{0}$, can be computed as,

$\mathbf{x}_{0 \alpha \beta}(n)=\mathbf{C} \cdot \mathbf{x}_{a b c}(n)$

then, an ideal $\mathbf{x}_{0 \alpha \beta, t}(n)$ is computed considering the actual position, 


$$
n_{0}=\left[\frac{f_{s}}{2 \pi 50} \cos ^{-1}\left(\frac{x_{\alpha}(n)}{\sqrt{x_{\alpha}^{2}(n)+x_{\beta}^{2}(n)}}\right)\right]
$$

where $\mathrm{n}_{0}$ is the integer part of the right side of (10). The first estimation of $\mathbf{x}_{0 \alpha \beta, t}(n)$ at $\mathrm{n}_{0}$ is then computed by,

$$
\mathbf{x}_{0 \alpha \beta, t}\left(n_{0}\right)=\left[\begin{array}{c}
x_{0}\left(n_{0}\right) \\
x_{\alpha}\left(n_{0}\right) \\
x_{\beta}\left(n_{0}\right)
\end{array}\right]=\left[\begin{array}{c}
0 \\
X \cos \left(n_{0} 2 \pi f_{s t}[n] T_{s}\right) \\
X \cos \left(n_{0} 2 \pi f_{s t}[n] T_{s}+\frac{\pi}{2}\right)
\end{array}\right]
$$

where $f_{s t}[n]$ is the estimated frequency. Its initial value can be 50 or $60 \mathrm{~Hz}$.

At $n_{0}+1$ the estimated position will be $\mathbf{x}_{0 \alpha \beta, t}\left(n_{0}+1\right)$. If the real frequency is higher than $50 \mathrm{~Hz}$ then the point $\mathbf{x}_{0 \alpha \beta, t}\left(n_{0}+1\right)$ lags behind $\mathbf{x}_{0 \alpha \beta}(n+1)$. The opposite is also true.

The new estimated value $f_{s t}[n+1]$ can be obtained using an iterative approach that minimize the estimation error using a PID controller, where

$$
f_{s t}[n+1]=f_{s t}[n]+\Delta f_{s t}[n]
$$

and

$$
\varepsilon[n]=\theta[n]-\theta_{t}[n]
$$

There are different approaches that can be followed in order to set the proportional $\left(\mathrm{k}_{\mathrm{p}}\right)$, integral $\left(\mathrm{k}_{\mathrm{i}}\right)$ and derivative $\left(\mathrm{k}_{\mathrm{d}}\right)$ constants. Table I summarizes typical values of the proportional, integral, and derivative feedback coefficients for PID-type controllers [4].

$$
\Delta f_{s t}[n]=\varepsilon[n] k_{p}+\frac{\varepsilon[n]-\varepsilon[n-1]}{T_{s}} k_{d}+\frac{T_{s}(\varepsilon[n]+\varepsilon[n-1])}{2} k_{i}
$$

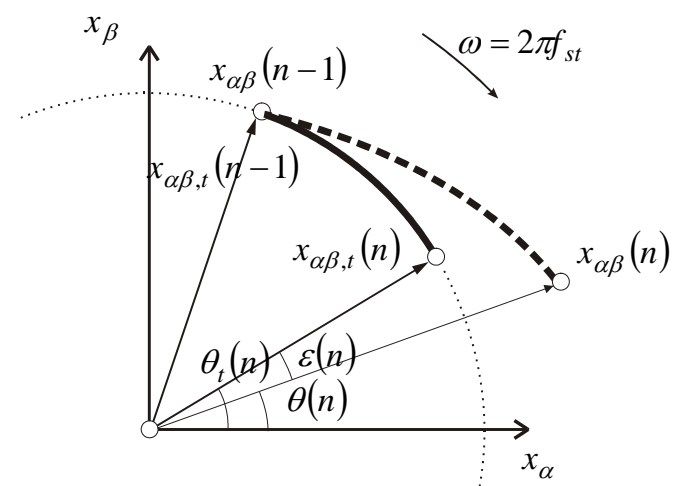

Fig. 2. Static reference frame representation with nonsinusoidal signals.
Table I. Typical values of the proportional, integral and derivative feedback coefficients for PID-type controllers [4].

\begin{tabular}{|l|ll|l|l|}
\hline Controller & $\mathrm{k}_{\mathrm{p}}$ & $\mathrm{k}_{\mathrm{i}}$ & $\mathrm{k}_{\mathrm{d}}$ \\
\hline PID & {$\left[\begin{array}{lll}0.1 & 0.5\end{array}\right]$} & {$\left[\begin{array}{lll}0.1 & 10\end{array}\right]$} & {$\left[\begin{array}{ll}0.05 & 1\end{array}\right]$} \\
& $\mathrm{k}_{\mathrm{p}, \max }$ & & $\mathrm{k}_{\mathrm{p}, \max } \mathrm{T}_{\text {osc }}$ & $\mathrm{k}_{\mathrm{p}, \max } \mathrm{T}_{\mathrm{osc}}$ \\
\hline
\end{tabular}

For $50 \mathrm{~Hz}$ systems, a good value of $\mathrm{k}_{\mathrm{pmax}}$ and $\mathrm{T}_{\text {osc }}$ can be 0.005 and 0.3 , respectively.

Figure 2 shows $\mathbf{x}_{0 \alpha \beta, t}(n)$ and $\mathbf{x}_{0 \alpha \beta}(n)$ considering that $\mathbf{x}_{0 \alpha \beta, t}(n)$ lags behind $\mathbf{x}_{0 \alpha \beta}(n)$.

Finally, the estimated frequency signal is low-pass filtered in order to reduce high-frequency oscillations.

\section{Test Results}

In order to put the technique to the test under dynamic conditions close to those that can be found in real power systems, some cases has been analyzed using two different approaches [5-13]: i) experimental test using an arbitrary three-phase voltage generator and ii) computational test using synthesized signals.

\section{A. Experimental Test Facility}

The laboratory test facility consists of a three-phase power supply that can generate arbitrary waveforms [14]. Figure 3 shows a block diagram of the test system facility.

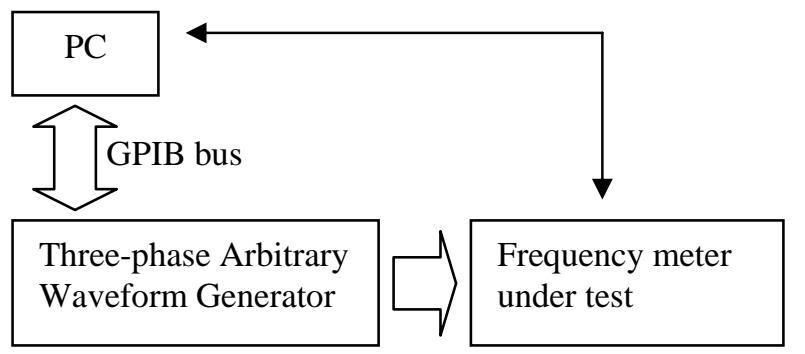

Fig. 3. Test system facility.

Figure 4 shows the estimation error of the algorithm with a $50 \mathrm{~Hz}$ signal disturbed with harmonic levels defined by table II.

Table II. Magnitude levels defined by UNE-EN 61000-4-13 for class 3 environments.

\begin{tabular}{|l|l|}
\hline $\mathrm{h}$ & $\mathrm{\%}_{1}$ \\
\hline 5 & 12 \\
7 & 10 \\
11 & 7 \\
13 & 7 \\
others & $<6$ \\
\hline
\end{tabular}


The percentages have been selected attending the standard UNE-EN 61000-4-13 [15] which defines the immunity tests to harmonics and interharmonics for industrial environment (class 3).

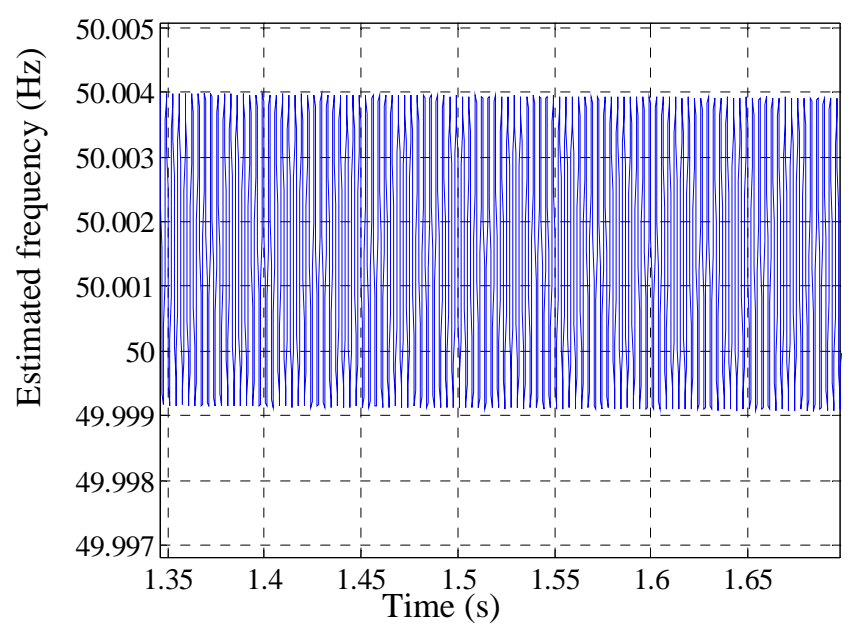

Fig. 4. Static test for a $50 \mathrm{~Hz}$ signal disturbed with class 3 maximum harmonic distortions.
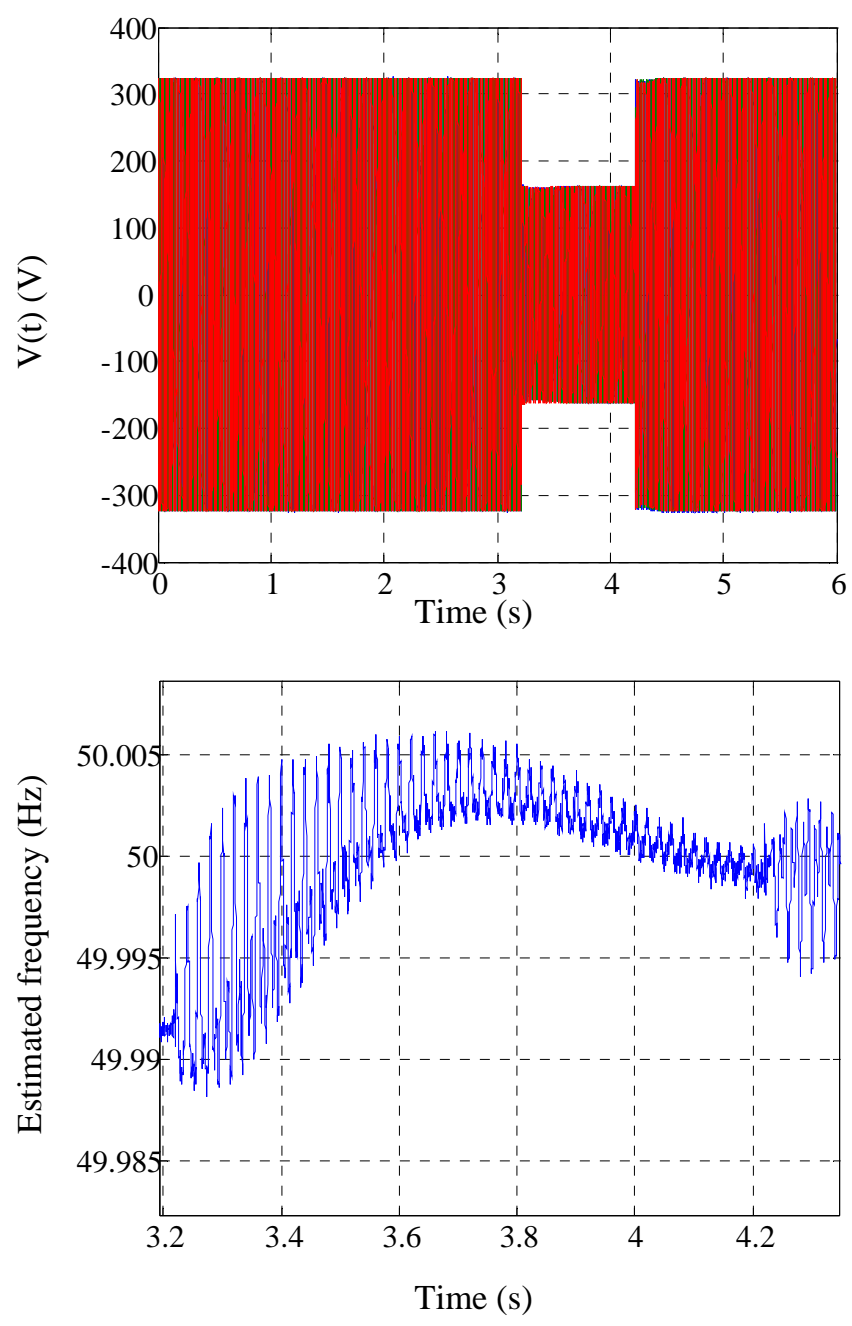

Fig. 5. Voltage and estimated frequency for a $50 \mathrm{~Hz}$ sinusoidal voltage disturbed with a $(50 \%, 1 \mathrm{~s})$ dip.
Dynamic test has been done in order to test the behavior of the algorithm to sags and small interruptions. This is a particularly severe test because one or two phases can reach values equal or close to zero.

Figure 5 shows the estimated frequency evolution under a $(50 \%, 1 \mathrm{~s})$ voltage sag which depth and duration is defined according to the values established by the standard UNE-EN 61000-4-11 [16] which defines the immunity test to voltage dips, short interruptions and voltage variations.

\section{B. Dynamic behavior with synthesized signals}

The performance of the algorithm under dynamic conditions using computational analysis has been done using synthesized signals.

A signal with a linear time varying frequency has been synthesized using MATLAB [17] according the expression (15), which represents a signal with their frequency varying from 45 to $65 \mathrm{~Hz}$ in half a second.

$$
f(n)=45+\frac{20}{0.5} n T_{s}
$$

Figure 6 shows the relative error of the estimated frequency.

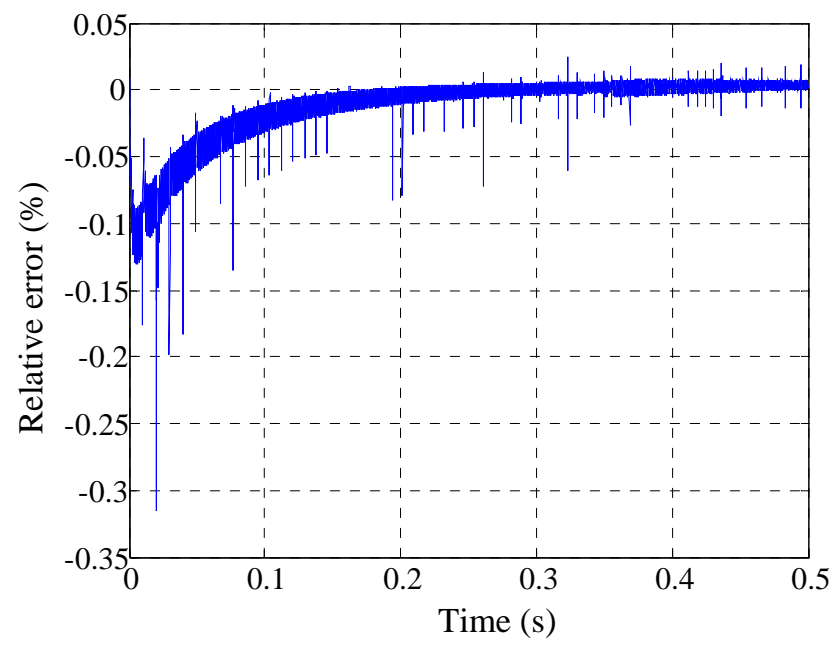

Fig. 6. Relative error for a signal with $f(t)=45+40 t$.

\section{Conclusion}

The proposed algorithm uses all available information of the three-phase voltage to estimate the value of the power system frequency. The digital filters are very simple to implement, and, since they are non-recursive, have no stability problems. The method has been tested with different conditions and has a good response in steady state and dynamic conditions. One of the aspects that can be improved is the initialization time and the output ripple. 
[9] Agrez, D.; "Frequency Estimation of the NonStationary Signals Using Interpolated DFT”. IEEE Instrumentation and Measurement Technology Conference. p- 925-930. 2002.

The authors would like to thank the support of the Spanish Government under the CICYT research project DPI2002-04416-C04-01.

\section{References}

[1] E. Clarke, Circuit analysis of a.c. power systems, Vol. I. Symmetrical and related components. New York. Wiley, 1943.

[2] R.H. Park, "Two-reaction theory of synchronous machines”, Pt. I. Transactions of the AIEE, 48, pp. 716-727, 1929.

[3] R.H. Park, "Two-reaction theory of synchronous machines”, Pt. II. Transactions of the AIEE, 52, pp. 352-355, 1933.

[4] Lyshevski, S.; Electromechanical Systems, Electric Machines, and Applied Mechatronics. CRC Press. 2000.

[5] Sezi, T., “A New Method for Measuring Power System Frequency”. IEEE Transmission and Distribution Conference. Vol. 1, p 400-405. 11-16 April 1999.

[6] Asnin, L.; Backmutsky, V.; Gankin, M.; “DSP methods for dynamic estimation of frequency and magnitude parameters in power systems transients”. IEEE Porto Power Tech Conference. 2001.

[7] Vainio, O.; Ovaska, S.; "Digital Filtering for Robust 50/60 Hz Zero-Crossing Detectors”. IEEE Transactions on Instrumentation and Measurement, Vol. 45, No. 2, p 426-430. April 1996.

[8] Yang, J.; Liu, C.; “A Precise Calculation of Power System Frequency”. IEEE Transactions on Power Delivery. Vol. 16, No. 3. p 361-366. July 2001. 